\title{
Clival osteomyelitis presenting as orbital apex syndrome
}

\author{
Irene Gil-Hernandez ${ }^{1 *}$, Amparo Lanuza-Garcia ${ }^{1}$, Raúl Martinez-Belda ${ }^{2}$ and Antonio Duch-Samper ${ }^{1,2}$ \\ ${ }^{1}$ Hospital Clínico Universitario de Valencia, Valencia, Spain \\ ${ }^{2}$ Universitat de València. Facultad de Medicina, Valencia, Spain
}

\begin{abstract}
A 73-year-old woman with Samter's triad presents a sudden onset of pain, ophthalmoplegia and decreased visual acuity of her right eye with RAPD. The clinical suspicion was an orbital apex syndrome. Radiological tests showed a massive occupation of sinus polyps that had led to osteomyelitis of the clivus. Emergency sinus surgery achieved resolution of the condition. This report details atypical skull base osteomyelitis presenting as an ophthalmologic emergency that could have been lethal.
\end{abstract}

\section{Introduction}

Orbital apex syndrome is an infrequent disease presenting as vision loss, pain and ophthalmoplegia due to affectation of II, III, IV, Va and VI cranial nerves at their entrance to the orbit. It is considered a real neuro-ophthalmologic emergency. The most common causes of orbital apex syndrome are inflammatory, infectious, tumoral, vascular and traumatic.

The authors report the case of a woman with a massive growth of sinus polyps that became infected, and whose contiguous spread caused osteomyelitis of the base of the skull presenting as orbital apex syndrome. This case report adhered to the ethical principles outlined in the Declaration of Helsinki as amended in 2013.

\section{Case report}

A 73-year-old woman presented with a 3-day history of progressive right retrobulbar pain and nose discharge, and a sudden onset diplopia and exophthalmos. Past medical history was significant for Samter's Triad and endoscopic sinus surgery 6 years before.

Exploration found visual acuity 20/40 OD and 20/20 OI. Right eye also had a relative afferent pupillary defect (RAPD), and partial ophthalmoplegia, more pronounced for adduction and abduction. Anterior segment examination and funduscopy were unremarkable. There was no loss of sensibility of facial skin. Laboratory examination showed a discrete elevation in Reactive Protein $(12.8 \mathrm{mg} / \mathrm{L})$ without leucocytosis.

An emergent angio-CT found no vascular abnormalities and cavernous sinus thrombosis was ruled out, but it showed massive occupation of frontal, maxilar and sphenoidal sinus with polyps (Figure 1A). A treatment with IV metilprednisolone $40 \mathrm{mg} / 12$ hours and IV ciprofloxacin $200 \mathrm{mg} / 12$ hours was started. Magnetic resonance with contrast demonstrated enhacement of clivus in its upper two thirds (Figure 2) and was diagnosed as clival osteomyelitis secondary to the polyposis. No collections were identified in cerebral parenchyma or arachnoid spaces. Cavernous sinuses were permeable without occupation or signs of thrombosis. Likewise, the internal carotid artery was permeable.
24 hours later the patient underwent endoscopic sphenoidotomy. Nose surgeons performed excision of nasal polyps and prior etmoidectomy enlargement. A biopsy specimen was obtained from the granulomatous content of sphenoidal sinus and its pathological analysis revealed changes from chronic rhinitis. No positive microbiological cultures were obtained. A CT scan with contrast taken 4 days after surgery demonstrated an increased aeration of sinuses and nostrils (Figure 1B). The patient presented favourable postoperative clinical evolution.

Two months later, her vision was $20 / 20$ in both eyes. Her right ocular motility was slightly restricted in abduction, without diplopia on primary position and she had no RAPD.
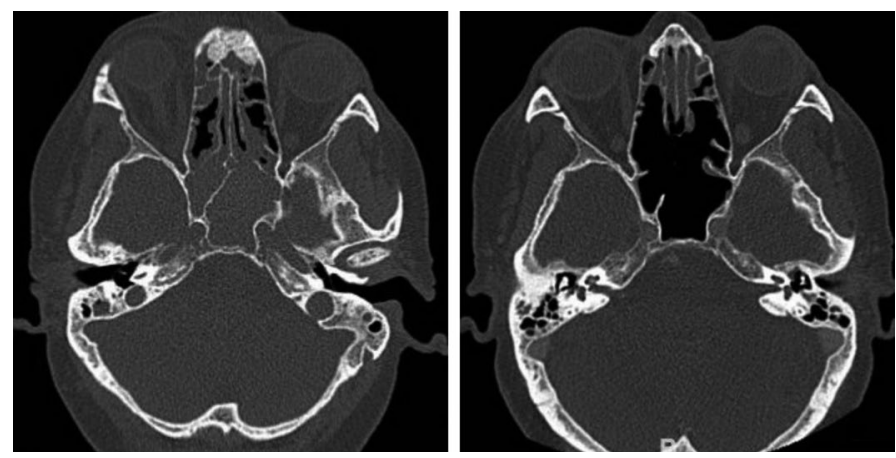

Figure 1. A) CT scan before surgery with massive polyp occupation of sphenoidal sinus. B) CT scan showing changes after polyp excision and sphenoidectomy

*Correspondence to: Irene Gil-Hernandez, Hospital Clínico Universitario de Valencia, Valencia, Spain, Tel: +34654938434, E-mail: igilher@gmail.com

Key words: orbital apex syndrome, skull base, clivus, osteomyelitis, polyps, samter's triad

Received: September 21, 2020; Accepted: October 12, 2020; Published: October 16,2020 


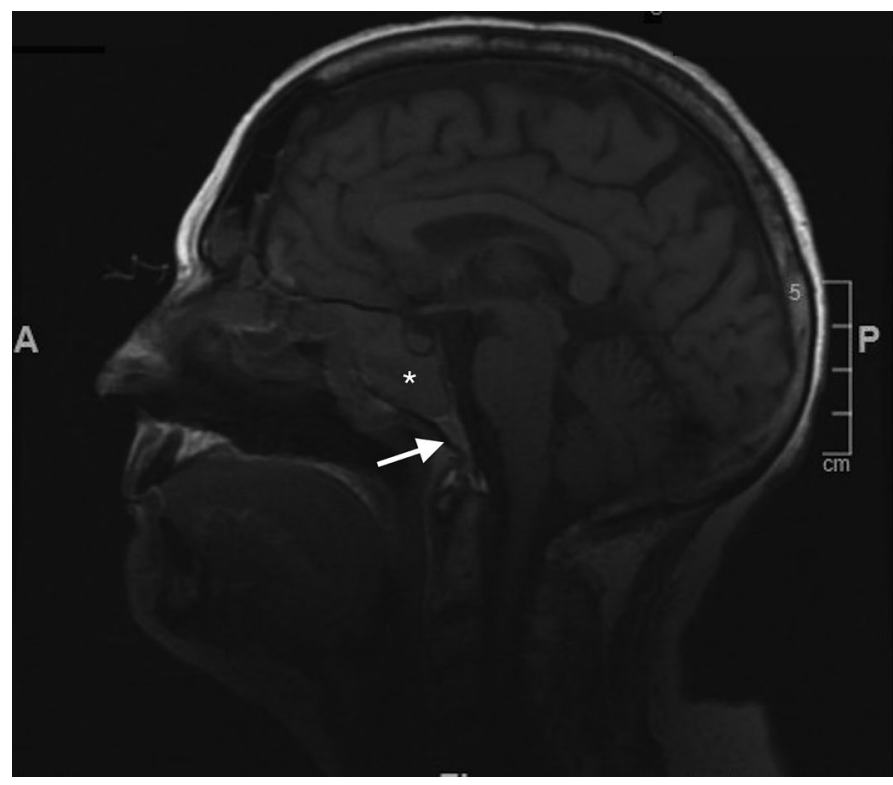

Figure 2. MR showing hipointensity of the clivus in its upper two thirds $(*)$ in contrast with normal signal (arrow)

\section{Discussion and conclusion}

Skull base osteomyelitis (SBO) is an uncommon condition, but it is potentially lethal [1]. Typical SBO affects temporal bone and it is generally a complication of otitis externa, most often seen in diabetic or immunocompromised adults [2]. Pseudomonas Aeruginosa is the most common pathogen [3]. SBO is defined as atypical or central when affecting sphenoid or occipital bone, usually involving the clivus [4]. It usually spreads from an infectious process involving the paranasal sinuses or adjacent bones of the skull base [5]. It can lead to complications such as venous sinus thrombosis, meningitis, or orbital apex syndrome [2]. Characteristic imaging findings are diffuse clival hypointensity on T1-weighted MR images due to bone marrow infiltration [5]. Its treatment is based on microbial culture of specimens. In our case the challenge was that the culture sample was negative. Surgery usually has a limited role, but in this case the removal of the polyps and the enlargement of the sphenoidectomy proved to be very effective. Compressive pathology of the optic nerve requires rapid intervention, and in this case the collaboration between radiologists, ENT surgeons and ophthalmologists allowed the resolution of the pathology.

\section{References}

1. Chandler J (1968) Malignant external Otitis. Laryngoscope 78: 1257-1294.

2. Johnson AK, Batra PS (2014) Central skull base osteomyelitis: An emerging clinical entity. Laryngoscope 124: 1083-1087. [Crossref]

3. Clark M, Pretorius P, Byren I, Milford C (2009) Central or Atypical Skull Base Osteomyelitis: Diagnosis and Treatment. Skull Base 19: 247-254. [Crossref]

4. Ridder GJ, Breunig C, Kaminsky J, Pfeiffer J (2015) Central skull base osteomyelitis new insights and implications for diagnosis and treatment. Eur Arch Otorhinolaryngol 272: 1269-1276. [Crossref]

5. Chang PC, Fischbein NJ, Holliday RA (2003) Central Skull Base Osteomyelitis in Patients without Otitis Externa: Imaging Findings. AJNR Am J Neuroradiol 24: 13101316. [Crossref]

Copyright: (C2020 Gil-Hernandez I. This is an open-access article distributed under the terms of the Creative Commons Attribution License, which permits unrestricted use, distribution, and reproduction in any medium, provided the original author and source are credited. 